\title{
Clinical follow-up of children with high vitamin B12 values: should we worry?
}

\author{
Davut Albayrak $^{1 \oplus}$, Canan Albayrak ${ }^{2 \oplus}$ \\ ${ }^{1}$ Department of Pediatric Hematology and Oncology, Medicalpark Samsun Hospital, Samsun; ${ }^{2}$ Department of Pediatric Hematology \\ and Oncology, Ondokuz Mayis University Faculty of Medi-cine, Samsun, Turkey.
}

\begin{abstract}
Background. Requests of Vitamin B12 test increased with the widespread use of autoanalysers. Although the cause of requests was deficiency suspicions, an important ratio of high levels of Vitamin B12 were reported to physicians by laboratory. Ratios of values of high Vitamin B12 among test request in adults are reported as 14$20 \%$ in present three monocentre studies and one multicentre study. There is no report on children with high vitamin B12 for both ratio in lab requests or clinical follow up.
\end{abstract}

Methods. We evaluated the records of 40 children (23 male /17 female) with high B12 values (>1000 pg/ $\mathrm{ml}$ ) retrospectively. Children were otherwise healthy children and were seen at outpatient pediatric clinics. Additionally, vitamin B12 values of 13 acute lymphoblastic leukemia patients at diagnosis time were retrieved to enlighten possible role of lymphocytes.

Result. Children did not have any malign or chronic diseases causing the high Vitamin B12 values. Holotranscobalamin levels were normal or slightly above. Two patients did develop leukemia later. Our follow up showed that high vitamin B12 values slightly decreased at 3 months and then remained unchanged later. The high numbers of $\mathrm{T}$ and $\mathrm{B}$ cells are not the source of vitamin B12 elevation.

Conclusions. Our study suggests that high-vitamin B12 values are usually benign in children but some patients may develop leukemia later. We suggest that patients should be followed up for some time after testing for severe hematological diseases.

Key words: vitamin B12, cobalamin, children, pediatric, holotranscobalamin.

Vitamin B12 (cobalamin, Cbl) is one of the most important vitamins that can be dissolved in water. It is absorbed in terminal ileum, transported by haptocorrins and stored in the liver. It is involved in methyl transfer and nucleotide synthesis. In children, deficiency leads to megaloblastic anemia and affects neurologic development. The importance of deficiency in children is well understood. There are many studies about vitamin B12 deficiency. ${ }^{1-3}$

The widespread use of autoanalysers in hospital laboratories has made vitamin B12 testing,

$凶$ Davut Albayrak

davutalb@omu.edu.tr

Received 11th May 2021, revised 30th May 2021, accepted 15th June 2021. like many tests, widespread, inexpensive, fast and accessible everywhere. Thus, all doctors, especially pediatricians, internist and neurologists, have started to request vitamin B12 tests from every patient in which the deficiency might have an impact or relation. Important data have accumulated in laboratory recording systems due to the increased requests. Analysis of these data showed that a significant proportion of the vitamin B12 tests were higher than normal. ${ }^{1-3}$ Thus, a new research area related to vitamin B12 levels was opened. The new questions are what is the meaning of this high vitamin B12 (hCbl) level found incidentally high in a patient and how to manage such a patient. These questions are very important, because the high levels of $\mathrm{Cbl}$ are seen in malign or proliferative diseases of myeloid and lymphoid 
series diseases such as promyelocytic leukemia, chronic myeloid leukemia (CML), autoimmune lymphoproliferative syndrome(ALPS), systemic inflammatory syndrome, polycythemia vera, hypereosinophilic syndrome, solid cancers, liver diseases such as acute hepatitis, cirrhosis, hepatocellular carcinoma, kidney disease, autoimmune disease and some infection diseases such as HIV and malaria. ${ }^{1-4}$

Elevation of $\mathrm{Cbl}$ may be one finding of these diseases or is likely to be the first presenting finding of a developing disease. This possibility makes it clinically important.

The ratio of hCbl among vitamin B12 samples that reached the laboratory is large enough to attract attention. Its ratio is $14 \%, 18.5 \%$ and $20 \%$ in three monocentre studies, and $18.5 \%$ a multicentre study. They also examined the relationship between high levels of $\mathrm{Cbl}$ with holotranscobalamin levels in the blood samples of these patients. They reported that $1 \%$ of samples are from patients with myeloproliferative disease..$^{4-7}$ However, there is no report on clinical follow up of adult or child patients with high vitamin B12 values.

The management of children with a hCbl value is still an important and unanswered question. There is no publication in children in terms of laboratory and clinical short and long-term follow-up. There is no report on clinical follow up of adult or child patients with hCbl values.

In this article, we present clinical and laboratory follow-up of patients who were referred to a pediatric hematology outpatient clinic by pediatricians because of vitamin B12 elevation. We also point to possible causes of $\mathrm{hCbl}$ and research subject areas for patients with $\mathrm{hCbl}$ levels.

\section{Material and Methods}

The records of 40 children with high $\mathrm{Cbl}$ value (>1000 pg/ml) were evaluated retrospectively. These patients were seen in our outpatient pediatric hematology clinic in the years of 2017-2020. Most of them were referred from other pediatric outpatient clinics for hematologic evaluation. The vitamin B12 levels had been requested during routine outpatient examinations for suspicion of deficiency. After history and physical examination, vitamin B12 (Normal value 200$800 \mathrm{pg} / \mathrm{ml}$ ), holotranscobalamin (Normal value 37-171 pmol/L), hemogram, ferritin, iron, ALT, AST, creatinine, folate values and abdominal ultrasonography of the patients were examined. They were also followed-up for any disease related with hCbl level such as myeloproliferative or lymphoproliferative disease. $\mathrm{Cbl}$ levels were measured initially and at third and sixth month. The patients were followed up clinically between 1 and 3 years.

In addition, vitamin B12 levels in 13 patients with acute lymphoblastic leukemia (ALL) having high vitamin B12 level at diagnosis were retrieved to discuss the possible lymphocyte origin of this high level.

Categorical data were expressed as number and percentage (\%). Scale variables were expressed as mean \pm standard deviation (SD) and range. Student's t test was used for comparison of means. Categorical variables were compared with Chi- Square. $\mathrm{P}<0.05$ was assessed as statistically significant. The statistical relationship between two variables was calculated by Pearson Correlation analysis.

\section{Results}

The records of 40 children with hCbl value $(>1000$ $\mathrm{pg} / \mathrm{ml}$ ) were evaluated retrospectively. Twenty three patients (58\%) were male. The average age of patients was $3.12 \pm 1.43$ year. Initial vitamin $\mathrm{B} 12$ are $1267,89 \pm 382,01 \mathrm{pg} / \mathrm{ml}$ and vitamin B12 in the third month are $1162.09 \pm 381.96 \mathrm{pg} / \mathrm{ml}$, in the sixth month are $1169.64 \pm 351.74 \mathrm{pg} / \mathrm{ml}$.

Demographic and laboratory findings are represented in Table I. Cbl levels decreased slightly in the third month. Difference of $\mathrm{Cbl}$ values between initial and in third month and sixth month was $\mathrm{p}=0.03$ and statistically significant. But difference of values between 
Table I. Demographic and laboratory findings of children with high Cobalamin $(\mathrm{Cbl})$ values.

\begin{tabular}{|c|c|c|c|}
\hline & Mean & SD & Range \\
\hline Male/Female & $23 / 17$ & & \\
\hline Age (year) & 3.18 & \pm 2.11 & $1-6.5$ \\
\hline Hemoglobin (g/dL) & 12.11 & \pm 0.88 & 9.6-13.6 \\
\hline White Blood Cell (x109/L) & 9.52 & \pm 3.53 & $3.75-15.49$ \\
\hline Lymphocyte (x109/L) & 4.63 & \pm 1.59 & $2.65-8.64$ \\
\hline C-reactive protein (mg/L) & 0.23 & \pm 0.4 & $0.01-1.39$ \\
\hline Ferritin (ng/ml) & 40.74 & \pm 17.6 & $9-77$ \\
\hline \multicolumn{4}{|l|}{ Holotranscobalamin (pmol/L) } \\
\hline (normal values 25-165 pmol/L) & 147.07 & \pm 60.06 & $82.80-630$ \\
\hline Initial Cbl (pg/ml) & 1267.89 & \pm 382.01 & $1000,9-2001$ \\
\hline At third month $\mathrm{Cbl}(\mathrm{pg} / \mathrm{ml})$ & 1162.09 & \pm 381.96 & $606.8-2000$ \\
\hline At sixth month $\mathrm{Cbl}(\mathrm{pg} / \mathrm{ml})$ & 1169.64 & \pm 351.74 & $466.2-2000$ \\
\hline
\end{tabular}

third and sixth month was not significant ( $p>0.05$ ). Holotranscobalamin levels are $147,07 \pm 60,06 \mathrm{pmol} / \mathrm{L}$ (normal values 25-165). Holotranscobalamin levels were normal \%67 and slightly above in $33 \%$. Correlations between vitamin $\mathrm{B} 12$ and holotranscobalamin levels was $\mathrm{r}=0.24$. There was no correlation between vitamin B12 and white blood cells, neutrophil, lymphocyte, platelet, hemoglobin and ferritin levels.

The follow-up period of the patients lasted from one year to three years. Two patients developed leukemia at follow-up. The first patient was a 2-year-old female patient and she developed ALL after two months of first admission. The second patient was a 6 year old male and he developed chronic myeloid leukemia (CML) after two years of the first hCbl level.

Characteristics of children with ALL are given in Table II. Cbl levels of ALL patients were normal range excluding one B cell ALL patient whose vitamin B12 level was 1154 pg/ml. In two patients with delta-gamma T cell ALL, Cbl levels were normal.

The research was reviewed and approved by institutional review board of Ondokuz Mayis University onclinicalstudieswithdate15/02/2021 and number B.30.2.ODM.0.20.08/26-61, and that participation involved informed consent. There is no financial assistance.
Table II. Characteristics of children with acute lymphoblastic leukemia.

\begin{tabular}{lc}
\hline Male/female & $6 / 7$ \\
T cell & 2 \\
B cell & 9 \\
deltagamma T cell & 2 \\
Mean cobalamin \pm SD $(\mathrm{pg} / \mathrm{ml})$ & $504.59 \pm 333.91$ \\
Median cobalamin, range $(\mathrm{pg} / \mathrm{ml})$ & $429.4(166.2-1154)$ \\
Mean White Blood Cell $\pm \mathrm{SD}$ & $30.35 \pm 52.57$ \\
$(x 109 / \mathrm{L})$ & \\
Median White Blood Cell, range & $10.9(1.03-184)$ \\
$(x 109 / \mathrm{L})$ & \\
\hline
\end{tabular}

\section{Discussion}

There is no report on clinical follow up of adult or child patients with $\mathrm{Cbl}$ values, although clinicians need to frequently decide about these patients. There is not enough publications about the meaning, importance, management and reasons of $\mathrm{hCbl}$ in a person who looks healthy in other respects and does not use vitamin B12 drugs. Our study is the first study to show what can be seen with clinical follow-up of patients. It has the potential to trigger other clinical studies.

Clinicians have been encountering more patients with $\mathrm{hCbl}$ results in the last decades. Patients and parents read the reasons for the $\mathrm{hCbl}$ from the internet and come to the physician with true or false information, fear and questions. Fear is due to the fact that the $\mathrm{hCbl}$ is associated 
with many hematological and chronic diseases such as juvenile myelomonocytic leukemia, CML, polycythemia vera, hypereosinophilic syndrome, chronic lymphocytic leukemia, acute leukemias, lymphomas, myelodysplastic leukemia, autoimmune lymphoproliferative syndrome, liver and kidney diseases. ${ }^{1-3}$ They want satisfying and detailed information. Patients live through a referral chain starting from their own doctor through a pediatrician to pediatric hematologist.

There have been many developments in recent years that have caused us to see more patients with hCbl. This increased our need for a management algorithm. We know better the relationship of $\mathrm{Cbl}$ deficiency and diseases. ${ }^{1-3,8}$ Ideas are being developed about how vitamin B12 deficiency changes the clinics of other diseases. Autoanalysers offer fast, inexpensive, and results available on the same day and then we can use these results to decide patient treatment. Doctors are very concerned about the consequences of $\mathrm{Cbl}$ deficiency and use auto analyzer facilities abundantly. They request measurement of plasma $\mathrm{Cbl}$ for their patients and intend to find a deficiency. But, see both high and low $\mathrm{Cbl}$ test results. ${ }^{4-7}$ Our cases are also determined by test request for deficiency suspicion.

To understand the cause, significance, possible side effects and possible new research areas of $\mathrm{Cbl}$ elevation, we must understand the structure, synthesis by bacteria and archea, transporting to human by food chain..$^{9-12}$

$\mathrm{Cbl}$ is a haptocorrin compound with cobalt in its center. Only a limited number of prokaryotes (some bacteria such as Pseudomonas denitrificans and archaea such as Thermosipho africanus) can synthesize cobalamins. These prokaryotes are a part of intestinal microbiata. Other living things take cobalamins from bacteria and archaea by a food chain. In the intestine, the synthesis of this molecule is by two pathways. ${ }^{13}$ The first path is anaerobic synthesis and studied in Salmonella typhimurium, the second pathway is aerobic synthesis and studied in Pseudomonas denitrificans..$^{14,15}$
If the bacteria in our gut synthesize $\mathrm{Cbl}$, why is $\mathrm{Cbl}$ deficiency occurring? $\mathrm{Cbl}$, are synthesized by the bacteria and archaea, but it remains in the living bacteria until it dies.

Most of bacteria are found in colons and it is after terminal ileum where the $\mathrm{Cbl}$ receptors are located. ${ }^{9-13}$ This is an important detail because the host may not absorbe $\mathrm{Cbl}$ in its own bacteria. For this reason, animals usually do not use these sources except that very small part.

In humans, $\mathrm{Cbl}$-containing proteins are hydrolyzed in the stomach and intestine. Free $\mathrm{Cbl}$ now binds to intrinsic factor secreted by parietal cells of the stomach. $\mathrm{Cbl}$ and intrinsic factor complex bind to cubulin receptors of epithelia of terminal ileum and enters epithelia cell by active transport by contributions of amnionless, megalin, serum paraoxonase/ arylesterase and receptor-associated-protein. ${ }^{1-4}$ In addition, $1 \%$ of an oral vitamin B12 is also passively absorbed. Then $\mathrm{Cbl}$ is secreted to portal vessels. In plasma, cobalamin is bound to either transcobalamin II (TC) as active form (holo-TC) or other haptocorrins transcobalamin $\mathrm{I}$, transcobalamin III ( $\mathrm{HC})$ as storage of $\mathrm{Cbl}$. holo-TC constitutes 6 to $20 \%$ of plasma Cbl but HC binds to $80-94 \%$ of them. Transcobalamin II is produced by mainly hepatocyctes and additionally enteric epithelia, endothelia and monocytes. Haptocorrins are a part of secondary granules. Filtered holotranscobalamin are reabsorbed by megalin receptors in renal tubules. Cells receive $\mathrm{Cbl}$ by holotranscobalamin II receptor CD 320. It is converted to methyl Cbl in cytoplasm and adenosyl $\mathrm{Cbl}$ in mitochondria. Methyl Cbl is a co-factor of methyl transferases, including methionine synthase (MetH) and corrinoid iron-sulfur protein (CFeSP). It is required for methionin synthesis from homosistein. Methionin is a methyl donor. In the methylation deficiency, thymidine synthesis and methylation of nucteotides are affected. $\mathrm{Cbl}$ deficiency also effect uracil synthesis. In bacteria, new molecules and enzymes are affected by methyl cobalamin levels. In mitochondria, Adenosyl cobalamin are required as a cofactor to convert methylmalonyl CoA to succinyl CoA. 
Adenosyl $\mathrm{Cbl}$ is involved to lipid metabolism by this way. ${ }^{9-12}$

Cbl supply to cell may be deficient despite $\mathrm{hCbl}$ levels, because HC has higher affinity than TC. If intestinal supply of $\mathrm{Cbl}$ is insufficient, present $\mathrm{Cbl}$ remain in the high affinity $\mathrm{HC}$ pool, low affinity TC cannot find sufficient vitamin B12 to bind. Low holoTC levels cause $\mathrm{Cbl}$ deficiency even in hCbl levels. ${ }^{9-12}$ Our cases have normal range holo-TC. There is no clinical deficiency. On the other hand holo-TC levels were normal although Cbl levels were high. That is, TC synthesis did not increase by some cause increasing HC levels

High levels of plasma $\mathrm{Cbl}$ in each disease are explained by different mechanisms..$^{9-12}$ Secretion of haptocorrin from leukocyte granules is responsible for high hCbl levels in lymphoprolipherative diseases, such as, automimmune lymphoproliperative disease (ALPS), myeloma and myeloprolipherative diseases such as polycytemia vera and other cancers. In autoimmune disease, hCbl levels are due to production from leukocytes, decreased uptake and decreased filtration by TC autoantibodies. In ALPS, Cbl level increases 15-20 fold of normal. In a study, HC is in lymphocyte lysate of ALPS-FAS subjects but not that of controls. TC is not present in lymphocytes of both groups. HC is present in granulocytes of both ALPS-FAS patients and controls. ${ }^{16}$ This also explains why there is no increase of vitamin B12 in lymphocytosis patients. Our acute lymphoblastic leukemia patients with $\mathrm{T}, \mathrm{B}$ and delta gamma $\mathrm{T}$ cells also show malign lymphocytes which were not usually the source of hCbl levels. Delta -gamma $\mathrm{T}$ cells have the same differences from alfa -beta T cells. Two delta gamma T cell leukemia/ lymphoma patients have normal values. We saw $\mathrm{hCbl}$ levels in myeloid leukemias such as JMML, CML and acute myeloid leukemias.

In kidney disease, high levels may be caused by a decreased renal $\mathrm{Cbl}$ clearance. In hepatic disease, release from lysed hepatocytes and decreased uptake by hepatocytes cause increased levels of TC. ${ }^{1-4}$
hCbl values in healthy children cannot be explained by suggested mechanisms $\mathrm{hCbl}$ associated disease. In our cases, kidney and liver diseases and abdominal tumors were excluded by abdominal ultrasonography and liver and kidney function test. Hematological disease was excluded by normal WBC, peripheral smear and clinical follow up. We need new explanations. For this reason, we look at the deficiency associated diseases and mechanism to see patterns and use it to explain the high levels in otherwise healthy children. The present list of variables are: oral intake by food or drugs, gastric intrinsic factor, consumption by intestinal parasites and bacterial overgrowth, bacterial flora, bacteriophages in flora, terminal ileum inflammation or resection, ph changes in gut, genetic disease of cubam receptor complex, transcobalamin II deficiency, haptocorrin releasing from leukocytes or malign cells, TC uptake by liver, TC reabsorption in kidney, holo-TC receptor deficiency and competition for limited $\mathrm{Cbl}$ between high affinity haptocorrins and TC. ${ }^{1-4}$ Now thinking about these variables: oral intake was excluded initially. Intrinsic factor does not rate limiting for absorption although low levels cause deficiency. Cubilin expression is rate-limiting step with $\mathrm{pH}$ dependency because 5 pg daily absorption is saturated amount of oral diet. ${ }^{17,18}$ Increasing expressions of absorption receptor complex may be related with high vitamin B12 levels. Mutation studies of these genes may give a relation.

The relationship between $\mathrm{Cbl}$ levels with genetic factors was investigated in deficient and normal persons. Heritability was estimated to be $59 \%$ in a study of monozygotic and dizygotic twins. This suggests that genetic effect is significant. ${ }^{19}$ Recent updates on whole genome SNP in deficient and control samples give a list of genes related to $\mathrm{Cbl}$ deficiency. ${ }^{20} \mathrm{~A}$ counterpart of these studies in patients with hCbl values may give a candidate gene list. Passive absorption rate is $1 \%$ of oral high doses and $500 \mathrm{mcg}$ give 5 pg of passive absorption..$^{21}$ But there was no oral b12 treatment in our patients. Dietary intake is also important. The mean serum $\mathrm{Cbl}$ in vegans 
was 33\% lower than in vegetarians and 57\% lower than in omnivores. ${ }^{22}$ Diets of our patients were typical children's diets.

In children with hCbl level, all known diseases should be excluded first by hematologic, biochemical and radiologic investigation. As in our cases, hCbl levels did not have any other abnormalities. In these patients, we should think of new causes increasing levels of $\mathrm{Cbl}$. One of them may be microbiota. ${ }^{23}$ Horses do not eat soil or meal but their vitamin B12 levels are normal. Their normal values are found to be associated with helicobacter colonization because helicobacter can synthetize $\mathrm{Cbl}$ and its upper intestinal colonization is appropriate for the absorption of vitamin B12 by dead bacteria. In humans helicobacter colonization is common and associated with peptic ulcus and antibiotic is used extensively. Does $\mathrm{Cbl}$ deficiency deepen after helicobacter therapy in a vegetarian? We did not know the role of helicobacter colonization in our patients. Another possibility is an increase of intestinal colonization. But there is opposite clinical finding present in intestinal duplication by $\mathrm{Cbl}$ consumption by increased bacterial colonization. Other possibilities are feeding habits may cause preferential increase of $\mathrm{Cbl}$ synthesis in bacteria and archaea by ingredients. Pickles, vinegar, butyrate foods may be examples of ingredient sources. ${ }^{23}$

In our study, leukemia developed in two of 40 children. The ratio 2/40 may be a coincidence. Annual risk of developing new leukemia in children is $4 / 100.000$ in the literature. ${ }^{24}$ That is $8 / 100000$ new cases in two years. The difference is significant when we compare $2 / 40$ and $8 / 100000$ with the chi-square test $(p=0,000)$. This important association should be validated with larger series.

We recommend that the following examinations be performed initially in a patient presenting with hCbl: WBC, peripheral smear, vitamin B12, holotranscobalamin, ferritin, folic acid, liver and kidney function tests and abdominal ultrasound. If there is cytopenia, tests should include direct coombs, autoantibodies and double negative ratio in alpha beta $T$ lymphocytes for ALPS and ALPS like diseases. In next visits, a limited test may be sufficient such as WBC, peripheral smear and Cbl. If there is a new abnormality in WBC or additional complaints, the test panel should be expanded appropriately. Patients should be followed up for two years for the development of leukemia and myeloproliferative disease. $\mathrm{hCbl}$ is not toxic. Meat restriction was not recommended because it is the main source of vitamin B12, iron and zinc. We are not afraid of $\mathrm{Cbl}$ elevation itself, but we do fear that it may be the first sign of a hematological disease such as leukemia.

Future clinical studies may be conducted in groups that can conduct laboratory research on pathways from oral intake to final clearance of $\mathrm{Cbl}$ and full genome analysis. This cooperation will provide sufficient material to find the causes leading to $\mathrm{hCbl}$ and to establish a cause and effect relationship.

In conclusion, a significant number of children seen in outpatient clinics due to other reasons have hCbl. Our study suggests a few patients may develop leukemia later. We suggest that patients should be followed up for two years after initial investigations for hematological, liver and kidney diseases showing hCbl levela. On this subject, longer and more detailed studies with larger case series are needed.

\section{Author contribution}

The authors confirm contribution to the paper as follows: study conception and design: DA; data collection: DA, CA; analysis and interpretation of results: DA, CA; draft manuscript preparation: DA, CA. All reviewed the results and approved the final version of the manuscript.

\section{Ethical approval}

The research was reviewed and approved by institutional review board of Ondokuz Mayis University on clinical studies 
with date 15/02/2021 and number B.30.2.ODM.0.20.08/26-61, and that participation involved informed consent.

\section{Source of funding}

There is no financial assistance.

\section{Conflict of interest}

The authors have no conflict of interest to inform.

\section{REFERENCES}

1. Langan RC, Goodbred AJ. Vitamin B12 deficiency: recognition and management. Am Fam Physician 2017; 96: 384-389.

2. Shipton MJ, Thachil J. Vitamin B12 deficiency-A 21st century perspective. Clin Med (Lond) 2015; 15: 145150. https://doi.org/10.7861/clinmedicine.15-2-145

3. Bridwell-Rabb J, Drennan CL. Vitamin B12 in the spotlight again. Curr Opin Chem Biol 2017; 37: 6370. https://doi.org/10.1016/j.cbpa.2017.01.013

4. Carmel R, Vasireddy H, Aurangzeb I, George K. High serum cobalamin levels in the clinical setting: clinical associations and holo-transcobalamin changes. Clin Lab Haematol 2001; 23: 365-371. https://doi.org/10.1046/j.1365-2257.2001.00134.x

5. Chiche L, Jean R, Romain F, et al. Clinical implications of high cobalamin blood levels for internal medicine. Rev Med Interne 2008; 29: 187-194. https://doi. org/10.1016/j.revmed.2007.07.007

6. Arendt JF, Nexo E. Cobalamin related parameters and disease patterns in patients with increased serum cobalamin levels. PLoS One 2012; 7: e45979. https://doi.org/10.1371/journal.pone.0045979

7. Brah S, Chiche L, Mancini J, Meunier B, Arlet JB. Characteristics of patients admitted to internal medicine departments with high serum cobalamin levels: results from a prospective cohort study. Eur J Intern Med 2014; 25: e57-e58. https://doi. org/10.1016/j.ejim.2014.01.014

8. Karakurt N, Albayrak C, Yener N, Albayrak D. Does vitamin B12 deficiency in infants cause severe clinical symptoms necessitating intensive care? J Pediatr Emerg Intensive Care Med 2019; 6: 134-139. https://doi.org/10.4274/cayd.galenos.2019.47568
9. Arendt JF, Nexo E. Unexpected high plasma cobalamin: proposal for a diagnostic strategy. Clin Chem Lab Med 2013; 51: 489-496. https://doi. org/10.1515/cclm-2012-0545

10. Andrès E, Serraj K, Zhu J, Vermorken AJ. The pathophysiology of elevated vitamin B12 in clinical practice. QJM 2013; 106: 505-515. https://doi. org/10.1093/qjmed/hct051

11. Serraj K, Mecili M, Housni I, Andrès E. Hypervitaminemia B12 (high level of cobalamin): physiopathology, role and interest in clinical practice. Presse Med 2011; 40(12 Pt 1): 1120-1127. https://doi.org/10.1016/j.lpm.2011.08.010

12. Ermens AA, Vlasveld LT, Lindemans J. Significance of elevated cobalamin (vitamin B12) levels in blood. Clin Biochem 2003; 36: 585-590. https://doi. org/10.1016/j.clinbiochem.2003.08.004

13. Fang H, Kang J, Zhang D. Microbial production of vitamin B12: a review and future perspectives. Microb Cell Fact 2017; 16: 15. https://doi.org/10.1186/ s12934-017-0631-y

14. Moore SJ, Warren MJ. The anaerobic biosynthesis of vitamin B12. Biochem Soc Trans 2012; 40: 581-586. https://doi.org/10.1042/BST20120066

15. Ainala SK, Somasundar A, Park S. Complete genome sequence of pseudomonas denitrificans ATCC 13867. Genome Announc 2013; 1: e00257-13. https:// doi.org/10.1128/genomeA.00257-13

16. Bowen RA, Dowdell KC, Dale JK, et al. Elevated Vitamin B12 levels in autoimmune lymphoproliferative syndrome attributable to elevated haptocorrin in lymphocytes. Clin Biochem 2012; 45: 490-492. https://doi.org/10.1016/j. clinbiochem.2012.01.016

17. Bor MV, Lydeking-Olsen E, Moller J, Nexo E. A daily intake of approximately 6 microg vitamin B-12 appears to saturate all the vitamin B-12-related variables in Danish postmenopausal women. Am J Clin Nutr 2006; 83: 52-58. https://doi.org/10.1093/ ajcn/83.1.52

18. Lam JR, Schneider JL, Zhao W, Corley DA. Proton pump inhibitor and histamine 2 receptor antagonist use and vitamin B12 deficiency. JAMA 2013; 310: 2435-2442. https://doi.org/10.1001/jama.2013.280490

19. Nilsson SE, Read S, Berg S, Johansson B. Heritabilities for fifteen routine biochemical values: findings in 215 Swedish twin pairs 82 years of age or older. Scand J Clin Lab Invest 2009; 69: 562-569. https://doi. org/10.1080/00365510902814646

20. Surendran S, Adaikalakoteswari A, Saravanan P, Shatwaan IA, Lovegrove JA, Vimaleswaran KS. An update on vitamin B12-related gene polymorphisms and B12 status. Genes Nutr 2018; 13: 2. https://doi. org/10.1186/s12263-018-0591-9 
21. Berlin H, Berlin R, Brante G. Oral treatment of pernicious anemia with high doses of vitamin B12 without intrinsic factor. Acta Med Scand 1968; 184: 247-258. https://doi.org/10.1111/j.0954-6820.1968. tb02452.x

22. Gilsing AM, Crowe FL, Lloyd-Wright Z, et al. Serum concentrations of vitamin B12 and folate in British male omnivores, vegetarians and vegans: results from a cross-sectional analysis of the EPIC-Oxford cohort study. Eur J Clin Nutr. 2010; 64: 933-939. https://doi.org/10.1038/ejcn.2010.142
23. Danchin A, Braham S. Coenzyme B12 synthesis as a baseline to study metabolite contribution of animal microbiota. Microb Biotechnol 2017; 10: 688-701. https://doi.org/10.1111/1751-7915.12722

24. Dong Y, Shi O, Zeng Q, Lu X, et al. Leukemia incidence trends at the global, regional, and national level between 1990 and 2017. Exp Hematol Oncol 2020; 9: 14. https://doi.org/10.1186/s40164-020-001706 